\title{
Clinical implication of serum CA125 for the prediction of malignancy in mucinous cystic neoplasms of the pancreas
}

\author{
SHENGMING DENG ${ }^{1-3^{*}}$, ZHIYAO FAN ${ }^{1-3^{*}}$, YITAO GONG $^{1-3^{*}}$, HE CHENG $^{1-3}$, \\ KAIZHOU JIN ${ }^{1-3}$, YUNZHEN QIAN ${ }^{1-3}$, ZHIWEN XIAO $^{1-3}$, YU LIU ${ }^{1-3}$, RUIJIE WANG $^{1-3}$, \\ YUWEI ZHENG $^{4}$, QUANXING NI ${ }^{1-3}$, XIANJUN YU $^{1-3}$, CHEN LIU $^{1-3}$ and GUOPEI LUO ${ }^{1-3}$ \\ ${ }^{1}$ Department of Pancreatic Surgery, Fudan University Shanghai Cancer Center; ${ }^{2}$ Department of Oncology, \\ Shanghai Medical College, Fudan University; ${ }^{3}$ Pancreatic Cancer Institute, Fudan University, Shanghai Pancreatic \\ Cancer Institute; ${ }^{4}$ Department of Pathology, Fudan University Shanghai Cancer Center, Shanghai 200032, P.R. China
}

Received September 15, 2020; Accepted October 6, 2020

DOI: $10.3892 /$ etm.2020.9287

\begin{abstract}
Mucinous cystic neoplasms (MCNs) of the pancreas have malignant potential. Carbohydrate antigen 125 (CA125) is a common widely used biomarker for cancers. However, the role of CA125 in predicting the malignant change of MCNs is currently unidentified. Patients with resected and pathologically confirmed MCN were identified from a prospectively maintained database. The predictive role of serum CA125 in assessing malignant change of MCNs was analyzed and compared with serum carbohydrate antigen 19-9 (CA19-9) and carcinoembryonic antigen (CEA). This study included 164 patients with MCN (low/moderate grade, 153 cases; high grade and invasive, 11 cases). The serum levels of CA125 in the high grade and invasive group $(45.1 \pm 42.1 \mathrm{U} / \mathrm{ml})$ was significantly higher than those in the low/moderate grade group $(21.0 \pm 46.2 \mathrm{U} / \mathrm{ml}, \mathrm{P}=0.006)$. The area under the receiver operating characteristic (ROC) curve of CA125 (0.75) for predicting malignancy of MCNs was higher than that of CA19-9 (0.68) or CEA (0.72). The prediction value of CA125 was improved when combined with CEA (CA125 alone, sensitivity $36.4 \%$, specificity $90.6 \%$, accuracy $86.6 \%$; combined with CEA, sensitivity $45.5 \%$, specificity $88.2 \%$, accuracy $85.0 \%$ ). It was concluded that serum CA125 shows value in predicting the malignant change of MCNs, especially when combined with serum CEA.
\end{abstract}

Correspondence to: Dr Guopei Luo or Dr Chen Liu, Department of Pancreatic Surgery, Fudan University Shanghai Cancer Center, 270 Dong'An Road, Xuhui, Shanghai 200032, P.R. China

E-mail: luoguopei@hotmail.com

E-mail: liuchen@fudanpci.org

*Contributed equally

Key words: CA125, CA19-9, CEA, mucinous cystic neoplasm, biomarker

\section{Introduction}

The mucinous cystic neoplasms (MCNs) of the pancreas are rare cystic tumors characterized by mucin production, presence of ovarian-type stroma, and malignant potential (1). It almost exclusively presents in middle-aged women and is located at the distal part of the pancreas (2). Unlike its mucinous producing counterpart intraductal papillary mucinous neoplasms (IPMNs), MCNs have no communication with either the main pancreatic duct or branch duct. Considering the possibility of malignant progression, surgical resection is usually recommended to MCNs $(3,4)$. The prognosis of patients with invasive MCNs is much better than that of patients with invasive IPMNs, with a 10-year disease-specific survival of 79.6 vs. $27.2 \%$, respectively (1).

Due to the invasive potential of MCNs, an accurate evaluation of malignant change is needed for appropriate clinical management (2). Variables including male sex, pancreatic head and neck location, increased tumor size, a solid component, and main pancreatic duct dilation have been reported to be positively associated with malignant change $(5,6)$. Carbohydrate antigen 125 (CA125), carbohydrate antigen 19-9 (CA19-9), and carcinoembryonic antigen (CEA) are commonly used biomarkers for various types of cancers. Serum CA19-9 and CEA have been recommended to the management of pancreatic cyst diseases $(4,7,8)$. However, the value of circulating CA19-9 and CEA in predicting MCN malignancy is limited. For example, a previous study showed that in 7 patients with pathologically confirmed severe dysplasia of mucinous cystic pancreatic neoplasms, 3 (42.9\%) patients had an elevated circulating CA19-9 and 2 (28.6\%) had an elevated circulating CEA (9). The study concluded that serum CA19-9 or CEA is not useful in screening mucinous cystic pancreatic neoplasm patients with malignancy (9). Although serum CA125 has been used in the management of various types of mucinous malignancies, its role in MCNs has not been identified.

In this study, by including 164 patients with resected and a histologic diagnosis of MCN, the predictive role of serum CA125 in assessing malignant change of MCNs was analyzed and compared with serum CA19-9 and CEA. In addition, the 
values of CA125 combined with serum CA19-9 or CEA in evaluating malignant alteration were also examined.

\section{Patients and methods}

Patients and data collection. All patients who underwent surgical resection of pancreatic MCN from May 2010 to November 2019 were identified from a prospectively maintained database of Fudan University Shanghai Cancer Center (Shanghai, China). All patients were pathologically verified to the diagnosis of MCN of the pancreas. Other pathological subtypes of pancreatic tumors including adenocarcinoma, serous cystic neoplasm, solid pseudopapillary tumor, and IPMN were excluded. Patients with previous or other concomitant cancer were also excluded. Clinicopathologic characteristics including age, sex, primary tumor location, cystic size, serum levels of CA19-9, serum levels of CEA, serum levels of CA125, and the operation type were collected. The recommended cutoff points based on the upper limit of the normal range were used (CA19-9, 37.0 U/ml; CA125, 35.0 U/ml; CEA, $5.2 \mathrm{ng} / \mathrm{ml})$, as used in previous studies $(8,10,11)$. This study was based on the ethical guidelines of the World Medical Association Declaration of Helsinki and was approved by the Ethics Committee of Fudan University Shanghai Cancer Center. All patients provided informed consent for the use of their personal data for research purposes.

Statistics. Variables were presented as median (range) or mean \pm standard deviation (SD), as appropriate. Comparisons of categorical variables were conducted with ranksum tests or Fisher's exact tests. Two-tailed t tests or ranksum test were used to compare parametric data. The ROC curve and the area under the ROC curve (AUC) were examined to determine the predictive value of biomarkers. Statistical analyses were conducted using Stata SE12.0 (StataCorp LP) and Prism statistical software (version 8; GraphPad Software, Inc.). $\mathrm{P}<0.05$ was considered to indicate a statistically significant difference.

\section{Results}

Baseline characteristics. The clinicopathologic characteristics of 164 patients with MCN are summarized in Table I. The female-to-male ratio was $11.6: 1$ and the median age was 48 years (range 18-82 years). Most of (91.5\%) the primary tumors were located at the body and tail of the pancreas. All the patients underwent tumor resection and $86.6 \%$ of patients received distal pancreatectomy. The mean cyst size in diameter was $4.6 \mathrm{~cm}$.

Characteristics according to malignancy. In this cohort, $6.7 \%$ $(11 / 164)$ of patents were diagnosed with malignant MCNs (high grade and invasive). The cystic size in the high grade and invasive group $(6.5 \pm 3.0 \mathrm{~cm})$ was larger than that in the low/moderate grade group $(4.5 \pm 2.8 \mathrm{~cm}, \mathrm{P}=0.017)$. There was no statistical difference between the low/moderate grade group and the high grade and invasive group in age $(\mathrm{P}=0.384)$, sex $(\mathrm{P}=0.192)$, primary tumor location $(\mathrm{P}=0.294)$, and operation type $(\mathrm{P}=0.609)$.

Tumor biomarker levels according to histological grade of dysplasia. Serum levels of CA19-9, CEA, and CA125 in the low/moderate grade group and the high grade and invasive group are shown in Table II and Fig. 1. The serum levels of CA19-9 (309.2 \pm 423.2 vs. $49.0 \pm 198.3 \mathrm{U} / \mathrm{ml}, \mathrm{P}=0.050)$, CEA (23.7 \pm 52.9 vs. $8.7 \pm 82.6 \mathrm{ng} / \mathrm{ml}, \mathrm{P}=0.018)$, and $\mathrm{CA} 125(45.1 \pm 42.1$ vs. $21.0 \pm 46.2 \mathrm{U} / \mathrm{ml}, \mathrm{P}=0.006$ ) in the high grade and invasive group was significantly higher than those in the low/moderate grade group. The area under the ROC curve of CA125 (0.75) for predicting malignancy of MCNs was higher than that of CA19-9 (0.68) or CEA (0.72, Fig. 2).

Combining serum biomarkers in predicting malignancy of MCNs. The sensitivity and specificity of CA125 as a biomarker in predicting malignancy of MCNs was $36.4 \%$ and $90.6 \%$, respectively, with an accuracy of $86.6 \%$ when using $35 \mathrm{U} / \mathrm{ml}$ as the cut-off value (Table III). The accuracy of CA125 in predicting malignancy of MCNs was higher than CA19-9 (78.6\%) and was comparable to CEA (91.7\%) when using the recommended cut-off values. The prediction value was improved when combining CA125 with CEA (sensitivity $45.5 \%$, specificity $88.2 \%$, accuracy $85.0 \%$ ).

\section{Discussion}

The role of CA125 in predicting the malignant change of MCNs is currently unknown. In this study, by including 164 patients with MCN (low/moderate grade, 153 cases; high grade and invasive, 11 cases), the serum levels of CA125 in the high grade and invasive group $(45.1 \pm 42.1 \mathrm{U} / \mathrm{ml})$ was significantly higher than those in the low/moderate grade group (21.0 $\pm 46.2 \mathrm{U} / \mathrm{ml}, \mathrm{P}=0.006)$. The area under the ROC curve of CA125 (0.75) for predicting malignancy of MCNs was higher than that of CA19-9 (0.68) or CEA (0.72). The predicting value of CA125 was improved when combined with CEA (CA125 alone, sensitivity $36.4 \%$, specificity $90.6 \%$, accuracy $86.6 \%$; combined with CEA, sensitivity $45.5 \%$, specificity $88.2 \%$, accuracy $85.0 \%$ ). These results indicate that CA125 could be used to predict the malignant change of MCNs and the predictive value is improved when combined with CEA.

CA125, also called MUC16, is a commonly used biomarker for various types of malignancy, especially ovarian tumors (12). The role of CA125 in MCNs has been largely unconfirmed. Nagashio et al (13) collected cyst fluid from 68 patients with pancreatic cystic diseases to determine the application of cyst fluid analysis (CA125, CEA, CA19-9, amylase, and cytology) in differentiating pancreatic cystic lesions. They showed that cyst fluid CA125 concentrations may help to segregate MCNs (median, $1135.5 \mathrm{U} / \mathrm{ml}$ ) from IPMNs $(2 \mathrm{U} / \mathrm{ml})$, serous cystic neoplasms $(68.5 \mathrm{U} / \mathrm{ml})$, and pseudocysts $(6.5 \mathrm{U} / \mathrm{ml})$, indicating the aberrant secretion of CA125 in MCNs (13). A multicentric retrospective study collected 347 patients with pancreatic MCN and found that CEA, CA19-9, and CA125 were remarkably different between the benign lesion group and the malignant lesion group $(\mathrm{P}<0.05)$ (14). However, none of these studies compared CA125 with CA19-9 or CEA. In this study, the area under the ROC curve of CA125 (0.75) for predicting malignancy of MCNs was higher than that of CA19-9 (0.68) or CEA (0.72), suggesting CA125 could be used to predict the malignant change of MCNs. Moreover, the predicting value of CA125 was improved when combined with CEA. 
Table I. Baseline characteristics.

\begin{tabular}{|c|c|c|c|c|}
\hline Characteristics & $\begin{array}{c}\text { Total } \\
(n=164)\end{array}$ & $\begin{array}{l}\text { Low/moderate grade } \\
\qquad(\mathrm{n}=153)\end{array}$ & $\begin{array}{l}\text { High grade and invasive } \\
\qquad(\mathrm{n}=11)\end{array}$ & P-value \\
\hline Age [median (range), years] & $48(18-82)$ & $48(18-75)$ & $58(28-82)$ & $0.384^{\mathrm{a}}$ \\
\hline Sex & & & & 0.192 \\
\hline Female $(\%)$ & $151(92.1)$ & $142(92.8)$ & $9(81.8)$ & \\
\hline Male $(\%)$ & $13(7.9)$ & $11(7.2)$ & $2(18.2)$ & \\
\hline Location & & & & 0.294 \\
\hline Head $(\%)$ & $14(8.5)$ & $14(9.2)$ & $0(0.0)$ & \\
\hline Body and tail (\%) & $150(91.5)$ & $139(90.8)$ & $11(100.0)$ & \\
\hline \multicolumn{5}{|l|}{ Operation type } \\
\hline Distal pancreatectomy & $142(86.6)$ & $131(85.6)$ & $11(100.0)$ & $0.609^{\mathrm{b}}$ \\
\hline Enucleation & $9(5.5)$ & $9(5.9)$ & $0(0.0)$ & \\
\hline Middle segmental pancreatectomy & $2(1.2)$ & $2(1.3)$ & $0(0.0)$ & \\
\hline Pancreatoduodenectomy & $11(6.7)$ & $11(7.2)$ & $0(0.0)$ & \\
\hline Cyst size (mean $\pm \mathrm{SD}, \mathrm{cm})$ & $4.6 \pm 2.9$ & $4.5 \pm 2.8$ & $6.5 \pm 3.0$ & $0.017^{\mathrm{a}}$ \\
\hline
\end{tabular}

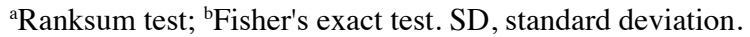

Table II. Tumor biomarker levels according to the histological grade of dysplasia.

\begin{tabular}{lccc}
\hline Biomarker & Total $(\mathrm{n}=164)$ & Low/moderate grade $(\mathrm{n}=153)$ & High grade and invasive $(\mathrm{n}=11)$ \\
\hline CA19-9 (mean $\pm \mathrm{SD}, \mathrm{U} / \mathrm{ml})$ & $67.0 \pm 228.7$ & $49.0 \pm 198.3$ & $309.2 \pm 423.2$ \\
$<37(\%)$ & $128(80.5)$ & $121(81.8)$ & $7(63.6)$ \\
$\geq 37(\%)$ & $31(19.5)$ & $27(18.2)$ & $4(36.4)$ \\
CEA $(\mathrm{mean} \pm \mathrm{SD}, \mathrm{ng} / \mathrm{ml})$ & $9.8 \pm 80.9$ & $8.7 \pm 82.6$ & $23.7 \pm 52.9$ \\
$<5.2(\%)$ & $149(94.9)$ & $141(96.6)$ & $8(72.7)$ \\
$\geq 5.2(\%)$ & $8(5.1)$ & $5(3.4)$ & $3(27.3)$ \\
CA125 $(\mathrm{mean} \pm \mathrm{SD}, \mathrm{U} / \mathrm{ml})$ & $22.8 \pm 46.2$ & $21.0 \pm 46.2$ & $45.1 \pm 42.1$ \\
$<35(\%)$ & $132(88.6)$ & $125(90.6)$ & $7(63.6)$ \\
$\geq 35(\%)$ & $17(11.4)$ & $13(9.4)$ & $4(36.4)$ \\
\hline
\end{tabular}

${ }^{a}$ Ranksum test. CA19-9, carbohydrate antigen 19-9; CEA, carcinoembryonic antigen; CA125, carbohydrate antigen 125.
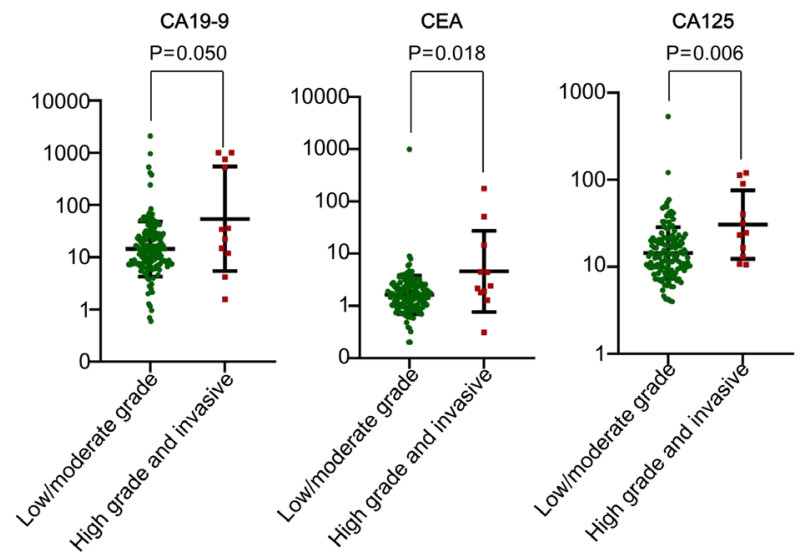

Figure 1. Individual serum levels of the three biomarkers (CA19-9, CEA CA125) in the low/moderate grade group and the high grade and invasive group of patients studied. P-values of the three biomarkers are also reported. CA19-9, carbohydrate antigen 19-9; CEA, carcinoembryonic antigen; CA125, carbohydrate antigen 125 .

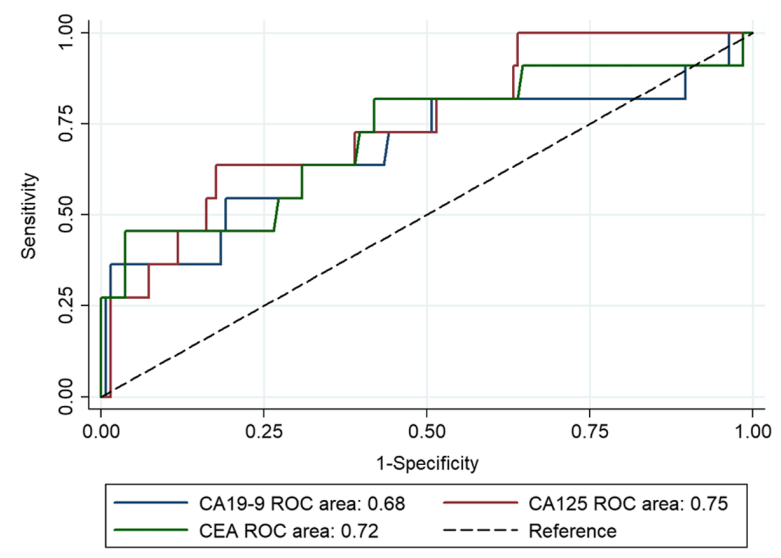

Figure 2. The ROC curve and area under the ROC curve for the three biomarkers (CA19-9, CEA, CA125) in predicting malignancy for MCNs. CA125 have higher prognostic value than other biomarkers. ROC, receiver operating characteristic; CA19-9, carbohydrate antigen 19-9; CEA, carcinoembryonic antigen; CA125, carbohydrate antigen 125; MCNs, mucinous cystic neoplasms. 
Table III. Diagnostic indices for CA19-9, CEA, and CA125 in predicting the malignant alteration of MCN.

\begin{tabular}{lcccccc}
\hline Value $^{\text {a }}$ & CA19-9 & CEA & CA125 & CA19-9 and/or CEA & CA19-9 and/or CA125 & CA125 and/or CEA \\
\hline Sensitivity (\%) & 36.4 & 27.3 & 36.4 & 54.5 & 54.5 & 45.5 \\
Specificity (\%) & 81.8 & 96.6 & 90.6 & 78.8 & 75.2 & 88.2 \\
PPV (\%) & 12.9 & 37.5 & 23.5 & 16.2 & 15.0 & 23.8 \\
NPV (\%) & 94.5 & 94.6 & 94.7 & 95.8 & 95.4 & 95.2 \\
Accuracy (\%) & 78.6 & 91.7 & 86.6 & 77.1 & 73.6 & 85.0 \\
\hline
\end{tabular}

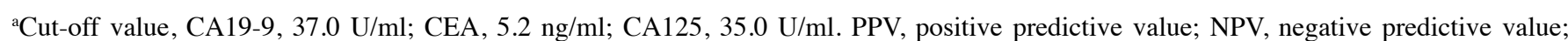
CA19-9, carbohydrate antigen 19-9; CEA, carcinoembryonic antigen; CA125, carbohydrate antigen 125; MCN, mucinous cystic neoplasm.

CA19-9, also termed as sialyl Lewis antigen a, is the best-validated biomarker for pancreatic adenocarcinoma $(10,15)$. The biosynthesis of CA19-9 is affected by the Lewis antigen status (15). Lewis antigen negative individuals, accounting for $5-10 \%$ of the population, have no or low secretion of CA19-9 (15). CA19-9 has been recommended to assess the malignant change of pancreatic cyst tumors, including IPMNs and MCNs $(3,5,7,16)$. Postlewait et al retrospectively identified 349 patients with MCN and demonstrated that patients with malignant neoplasms had a higher serum CA19-9 level than those without (median, 210 vs. $15 \mathrm{U} / \mathrm{ml}, \mathrm{P}=0.001$ ) (5). Zhao et al (17) showed that serum CA19-9 levels were significantly higher in malignant MCNs than in benign MCNs patients $(\mathrm{P}=0.026)$ for resected MCNs. Our study is consistent with previous findings, which finds that the serum levels of CA19-9 in the high grade and invasive group $(309.2 \pm 423.2 \mathrm{U} / \mathrm{ml})$ was significantly higher than those in the low/moderate grade group $(49.0 \pm 198.3 \mathrm{U} / \mathrm{ml}, \mathrm{P}=0.050)$, with an area under the ROC curve of 0.68 .

CEA is a glycosylation biomarker which has been widely applied in the management of gastrointestinal malignancies (10). For pancreatic cyst tumors, cyst fluid CEA have been used to differentiate mucinous tumors $(>192 \mathrm{ng} / \mathrm{ml})$ from non-mucinous tumors $(<5 \mathrm{ng} / \mathrm{ml})(4,18)$. Brugge et al (19) showed cyst fluid CEA (optimal cut-off of $192 \mathrm{ng} / \mathrm{ml}$, accuracy 79\%) to be more useful than endoscopic ultrasound morphology (accuracy 51\%) or cyst fluid cytology (accuracy 59\%) for differentiating mucinous from nonmucinous cystic lesions. However, cyst fluid CEA has limited role in predicting malignancy or radiographic progression mucinous cysts of the pancreas (18). Several studies have also determined circulating CEA in predicting malignant MCNs $(14,16,17)$. For example, Zhao et al (17) demonstrated that serum CEA levels were significantly higher in malignant MCNs than in benign MCNs patients $(\mathrm{P}=0.005)$ for 82 patients with resected MCNs. In this study, the serum levels of CEA in the high grade and invasive group $(23.7 \pm 52.9 \mathrm{ng} / \mathrm{ml})$ was significantly higher than those in the low/moderate grade group $(8.7 \pm 82.6 \mathrm{ng} / \mathrm{ml}, \mathrm{P}=0.018)$, with an area under the $\mathrm{ROC}$ curve of 0.72 . Our study confirms previous findings that CEA could be used to predict malignant MCNs.

In summary, CA125 shows promising value in predicting malignant MCNs. Its predictive value may be strengthened by combining with CEA. However, further evidence, especially evidence from multicentric, large sample size, and/or prospective studies is needed to confirm the findings.

\section{Acknowledgments}

Not applicable.

\section{Funding}

This work was supported by the National Natural Science Foundation of China (grant nos. 81625016, 81871940 and 81902417), the Scientific Innovation Project of Shanghai Education Committee (grant no. 2019-01-07-00-07-E00057), Clinical and Scientific Innovation Project of Shanghai Hospital Development Center (SHDC12018109), the Shanghai Natural Science Foundation (grant no. 17ZR1406300), the Shanghai Cancer Center Foundation for Distinguished Young Scholars (grant no. YJJQ201803), and the Fudan University Personalized Project for 'Double Top' Original Research (grant no. XM03190633).

\section{Availability of data and materials}

The datasets used and/or analyzed during the current study are available from the corresponding author on reasonable request.

\section{Authors' contributions}

GL, CL, XY designed the study. SD, ZF, YG, HC, KJ, YQ, ZX, YL, RW, YZ, QN, XY, CL and GL collected and analyzed data. GL wrote the draft. XY and CL revised it critically. All authors read and approved the final manuscript.

\section{Ethics approval and consent to participate}

The study protocol was approved by the ethics committee of Fudan University Shanghai Cancer Center.

\section{Patient consent for publication}

Written informed consent was acquired from all of the subjects enrolled.

\section{Competing interests}

The authors declare that they have no competing interests. 


\section{References}

1. Griffin JF, Page AJ, Samaha GJ, Christopher A, Bhaijee F, Pezhouh MK, Peters NA, Hruban RH, He J, Makary MA, et al: Patients with a resected pancreatic mucinous cystic neoplasm have a better prognosis than patients with an intraductal papillary mucinous neoplasm: A large single institution series. Pancreatology 17: 490-496, 2017.

2. Nilsson LN, Keane MG, Shamali A, Millastre Bocos J Marijinissen van Zanten M, Antila A, Verdejo Gil C, Del Chiaro $M$ and Laukkarinen J: Nature and management of pancreatic mucinous cystic neoplasm (MCN): A systematic review of the literature. Pancreatology 16: 1028-1036, 2016.

3. Tanaka M, Fernández-Del Castillo C, Kamisawa T, Jang JY, Levy P, Ohtsuka T, Salvia R, Shimizu Y, Tada M and Wolfgang CL: Revisions of international consensus Fukuoka guidelines for the management of IPMN of the pancreas. Pancreatology 17: 738-753, 2017.

4. Stark A, Donahue TR, Reber HA and Hines OJ: Pancreatic cyst disease: A Review. JAMA 315: 1882-1893, 2016.

5. Postlewait LM, Ethun CG, McInnis MR, Merchant N, Parikh A, Idrees K, Isom CA, Hawkins W, Fields RC, Strand M, et al: Association of preoperative risk factors with malignancy in pancreatic mucinous cystic neoplasms: a multicenter study. JAMA Surg 152: 19-25, 2017.

6. Le Baleur Y, Couvelard A, Vullierme MP, Sauvanet A, Hammel P, Rebours V, Maire F, Hentic O, Aubert A, Ruszniewski P, et al: Mucinous cystic neoplasms of the pancreas: Definition of preoperative imaging criteria for high-risk lesions. Pancreatology 11: 495-499, 2011.

7. European Study Group on Cystic Tumours of the Pancreas: European evidence-based guidelines on pancreatic cystic neoplasms. Gut 67: 789-804, 2018.

8. Fritz S, Hackert T, Hinz U, Hartwig W, Büchler MW and Werner J: Role of serum carbohydrate antigen 19-9 and carcinoembryonic antigen in distinguishing between benign and invasive intraductal papillary mucinous neoplasm of the pancreas. Br J Surg 98: 104-110, 2011.

9. Pezzilli R, Calculli L, Melzi d'Eril G and Barassi A: Serum tumor markers not useful in screening patients with pancreatic mucinous cystic lesions associated with malignant changes. Hepatobiliary Pancreat Dis Int 15: 553-557, 2016.

10. Luo G, Liu C, Guo M, Cheng H, Lu Y, Jin K, Liu L, Long J, Xu J, $\mathrm{Lu} \mathrm{R}$, et al: Potential biomarkers in lewis negative patients with pancreatic cancer. Ann Surg 265: 800-805, 2017.

11. Liu L, Xu H, Wang W, Wu C, Chen Y, Yang J, Cen P, Xu J, Liu C, Long J, et al: A preoperative serum signature of CEA+/CA125+/CA19-9 $\geq 1000 \mathrm{U} / \mathrm{ml}$ indicates poor outcome to pancreatectomy for pancreatic cancer. Int J Cancer 136 2216-2227, 2015.
12. Felder M, Kapur A, Gonzalez-Bosquet J, Horibata S, Heintz J, Albrecht R, Fass L, Kaur J, Hu K, Shojaei H, et al: MUC16 (CA125): Tumor biomarker to cancer therapy, a work in progress. Mol Cancer 13: 129, 2014.

13. Nagashio Y, Hijioka S, Mizuno N, Hara K, Imaoka H, Bhatia V, Niwa Y, Tajika M, Tanaka T, Ishihara M, et al: Combination of cyst fluid CEA and CA 125 is an accurate diagnostic tool for differentiating mucinous cystic neoplasms from intraductal papillary mucinous neoplasms. Pancreatology 14: 503-509, 2014.

14. Li WL, Xu YD, Han X, Wu WC and Lou WH; Pancreatic Surgery of Chinese Academic Society of Young Surgeons: Clinical analysis and prognosis factors of malignancy in the patients with mucinous cystic neoplasms of the pancreas. Zhonghua Wai Ke Za Zhi 58: 225-229, 2020 (In Chinese).

15. Luo G, Fan Z, Cheng H, Jin K, Guo M, Lu Y, Yang C, Fan K, Huang Q, Long J, et al: New observations on the utility of CA19-9 as a biomarker in Lewis negative patients with pancreatic cancer. Pancreatology 18: 971-976, 2018.

16. Goh BK, Tan DM, Thng CH, Lee SY, Low AS, Chan CY, Wong JS, Lee VT, Cheow PC, Chow PK, et al: Are the Sendai and Fukuoka consensus guidelines for cystic mucinous neoplasms of the pancreas useful in the initial triage of all suspected pancreatic cystic neoplasms? A single-institution experience with 317 surgically-treated patients. Ann Surg Oncol 21: 1919-1926, 2014.

17. Zhao ZM, Jiang N, Gao YX, Yin ZZ, Zhao GD, Tan XL, Xu Y and Liu R: Clinical diagnosis and management of pancreatic mucinous cystadenoma and cystadenocarcinoma: Single-center experience with 82 patients. World J Gastrointest Oncol 12: 642-650, 2020

18. Nagula S, Kennedy T, Schattner MA, Brennan MF, Gerdes H, Markowitz AJ, Tang L and Allen PJ: Evaluation of cyst fluid CEA analysis in the diagnosis of mucinous cysts of the pancreas. J Gastrointest Surg 14: 1997-2003, 2010.

19. Brugge WR,LewandrowskiK,Lee-LewandrowskiE,CentenoBA, Szydlo T, Regan S, del Castillo CF and Warshaw AL: Diagnosis of pancreatic cystic neoplasms: A report of the cooperative pancreatic cyst study. Gastroenterology 126: 1330-1336, 2004.

This work is licensed under a Creative Commons Attribution-NonCommercial-NoDerivatives 4.0 International (CC BY-NC-ND 4.0) License. 